\title{
The impact of high co-expression of Sp1 and HIF1 $\alpha$ on prognosis of patients with hepatocellular cancer
}

\author{
LIANG LIU ${ }^{1}$, PING JI ${ }^{2}$, NING QU ${ }^{3}$, WEI-LIN PU ${ }^{4}$, DAO-WEN JIANG ${ }^{5}$, \\ WEI-YAN LIU ${ }^{5}$, YA-QI LI ${ }^{6}$ and RONG-LIANG SHI ${ }^{3,5}$ \\ ${ }^{1}$ Department of Oncology, Fudan University Shanghai Cancer Center, Fudan University, Shanghai 200032; \\ ${ }^{2}$ Shanghai Public Health Clinical Center, Key Laboratory of Medical Molecular Virology of MOE/MOH, \\ Fudan University, Shanghai 201508; ${ }^{3}$ Department of Head and Neck Surgery, Fudan University Shanghai Cancer Center, \\ Fudan University, Shanghai 200032; ${ }^{4}$ Ministry of Education Key Laboratory of Contemporary Anthropology and State Key \\ Laboratory of Genetic Engineering, Fudan University, Shanghai 200433; ${ }^{5}$ Department of General Surgery, \\ Minhang Hospital, Fudan University, Shanghai 201199; ${ }^{6}$ Department of Colorectal Surgery, \\ Fudan University Shanghai Cancer Center, Fudan University, Shanghai 200032, P.R. China
}

Received November 24, 2015; Accepted April 12, 2016

DOI: $10.3892 / \mathrm{ol} .2016 .4634$

\begin{abstract}
Transcription factor specificity protein 1 (Sp1) and hypoxia-inducible factor $1 \alpha(\mathrm{HIF} 1 \alpha)$ serve vital roles in tumor growth and metastasis. The present study aimed to evaluate the impact of co-expression of Sp1 and HIF1 $\alpha$ on the prognosis of patients with hepatocellular cancer (HCC) using The Cancer Genome Atlas (TCGA) database and to validate the association between the expression levels of Sp1/HIF1 $\alpha$ in HCC specimens and patient survival using immunohistochemical analysis. A total of 214 eligible patients with HCC from TCGA database were collected for the study. The expression profile of $\mathrm{Spl}$ and HIF1 $\alpha$ were obtained from the TCGA RNAseq database. Clinicopathological characteristics, including age, height, weight, gender, race, ethnicity, family cancer history, serum $\alpha$-fetoprotein (AFP), surgical procedures and TNM stage were collected. The Cox proportional hazards regression model and Kaplan-Meier curves were
\end{abstract}

Correspondence to: Dr Ya-Qi Li, Department of Colorectal Surgery, Fudan University Shanghai Cancer Center, Fudan University, 270 Dong'an Road, Shanghai 200032, P.R. China E-mail: liyaqifudan@sina.com

Professor Rong-Liang Shi, Department of Head and Neck Surgery, Fudan University Shanghai Cancer Center, Fudan University, 270 Dong'an Road, Shanghai 200032, P.R. China

E-mail: docshawn@163.com

Abbreviations: HCC, hepatocellular cancer; Sp1, transcription factor specificity protein 1 ; HIF1 $\alpha$, hypoxia-inducible factor $1 \alpha$; TCGA, The Cancer Genome Atlas; AFP, $\alpha$-fetoprotein; IHC, immunohistochemistry; TNM, tumor-node-metastasis; AJCC, American Joint Committee on Cancer; CSS, cancer-specific survival; HR, hazard ratio; CI, confidence interval

Key words: hepatocellular cancer, Sp1, HIF1 $\alpha$, The Cancer Genome Atlas, prognosis used to assess the relative factors. Receiver operating characteristic (ROC) curves for cancer-specific survival (CSS) prediction were plotted to compare the prediction ability of expression of Sp1 and HIF1 $\alpha$ and their co-expression. The location and expression of Sp1 and HIF1 $\alpha$ in the HCC tissues were detected by immunohistochemistry (IHC) to verify the association between these two genes and CSS. The results demonstrated that the expressions of Sp1 and HIF1 $\alpha$ were significantly increased in the succumbed group $(\mathrm{P}=0.001)$, compared with the surviving group. The CSS rates were $60.1 \%$ at 3 years (1,067 days), $35.8 \%$ at 5 years (1,823 days) and $9.5 \%$ at 10 years $(3,528$ days). Multivariate Cox regression analysis demonstrated that only the high expression levels of Sp1 and HIF1 $\alpha\left(\geq 2 \times 10^{3}\right)$ were independent predictors for cancer mortality, with $\mathrm{P}=0.001$ and $\mathrm{P}=0.029$, respectively. The area under the curve for the ROC was found to be higher using the combination testing for two genes (0.751) in predicting cancer mortality, compared to a single gene ( 0.632 for Sp1 and 0.717 for HIF1 $\alpha$ ). Based on the cutoff points for gene expression, patients were divided into 3 groups: G1 (both genes $<2 \times 10^{3}$ ), $\mathrm{G} 2$ (either gene $\geq 2 \times 10^{3}$ ) and $\mathrm{G} 3$ (both genes $\geq 2 \times 10^{3}$ ). The risk of cancer mortality increased with high expression of genes, and G3 exhibited a greater risk than G2 when compared with the $\mathrm{G} 1$ group $(\mathrm{HR}=5.420,95 \%$ CI 2.767-10.616, $\mathrm{P}=0.001$; $\mathrm{HR}=3.270,95 \%$ CI 1.843-5.803, $\mathrm{P}=0.001)$. The IHC staining results indicated that patients who died of cancer presented with significantly higher expression levels of these genes compared with those that did not $(\mathrm{P}=0.001)$. In summary, high expression levels of Sp1 and HIF1 $\alpha$ in HCC tissues were associated with poor prognosis; in particular, the co-expression of these two genes increased the risk of cancer mortality.

\section{Introduction}

Hepatocellular cancer (HCC) is one of the most common malignant tumors with an annually increasing incidence and mortality rate in China (1). Approximately 394,770 estimated 
liver cancer cases were newly diagnosed in 2012, and the number continues to increase annually (1). Patients diagnosed with HCC typically present with tumors that are more aggressive and advanced, and they also present with more frequent distant metastases. Although surgical resection and liver transplantation provide a certain chance to cure it, the prognosis of HCC remains unfavorable due to the high risk of recurrence and distant metastasis postoperatively (2). Therefore, it is important to find convincing molecular markers that correlate with poor prognosis to tailor the treatment for the high-risk patients.

Transcription factor specificity protein $1(\mathrm{Sp} 1)$ is a sequence specific DNA binding protein, which has been reported to be abnormally expressed and activated in tumor tissues in a previous study (3). Sp1 is involved in the regulation of tumor cell proliferation, invasion, angiogenesis, and other biological functions $(3,4)$. Recently, Sp1 has been proposed as an indicator for poor prognosis in patients with gastric, pancreatic, breast and thyroid tumor cells in certain studies (5-7). Meanwhile, hypoxia-inducible factor 1 (HIF1), composed of one $\alpha$ and one $\beta$ subunit, has been suggested to be an important molecule in cell response to hypoxia involving in the regulation of angiogenesis, cell adhesion, energy metabolism, and apoptosis (8). HIF1-inducing angiogenesis serves an important role in tumor growth (8); HIF1 expression is elevated in HCC tissues and highly associated with poor prognosis (9). However, the combined expression of Spl and HIF1 in HCC and its impact on prognosis have not previously been reported in HCC.

The present study aimed to evaluate whether the expression levels of Sp1 and HIF1 are correlated with each other and other clinicopathological factors using The Cancer Genome Atlas (TCGA) database, and to investigate the prognostic impact of combination testing in HCC.

\section{Materials and methods}

Patients and samples. The data files were used to analyze Sp1 and HIF1 expression, and the level 3 clinical data of 351 HCC patients were initially downloaded from the TCGA (http://cancergenome.nih.gov/) data portal website, by using the data matrix link to access RNASeq data for liver HCC dataset and by using the UNC (IlluminaHiSeq_RNAseqV2) data platform. The reads per kilobase per million mapped reads value was used to represent the expression level of each gene. The gene expression data above for each individual TCGA sample with hepatic cancer during 1996-2013 were organized into an Excel file [system object model (SOM) file, labeled SOM Butler complete gene express set]. Samples were excluded for any of the following reasons: If they possessed in situ or incomplete tumor-node-metastasis (TNM) staging, no evaluation on lymph nodes or differentiation grade or histological type pathologically, died within 10 days following surgery, or had multiple primary malignant neoplasms as determined by Extent of Disease Codes (10). Clinicopathological parameters, including age, height, weight, sex, race, ethnicity, relative family cancer history, serum $\alpha$-fetoprotein (AFP), surgical procedures, and TNM stage were also assessed. These data were then used to determine the correlations between HIF1 $\alpha$, Sp1 RNA levels and prognosis, as indicated in the results. The guidance of the
2010 TNM classification of American Joint Committee on Cancer (AJCC)/International Union Against Cancer $(11,12)$ was followed for postoperative evaluation of staging.

The paraffin-embedded HCC specimens of 50 patients (25 succumbed to hepatic cancer and 25 survived in the follow-up period) who underwent a surgical procedure were obtained form Fudan University Shanghai Cancer Center in Shanghai, China. All cases were histologically confirmed. Patients were regularly followed up every 3-6 months. Events, such as tumor recurrence, progression, metastasis, and mortality, were recorded. The study was approved by Ethics Committee of Fudan University Shanghai Cancer Center, and written informed consent was obtained from all patients.

Immunohistochemical staining (IHC). IHC was performed according to a standard protocol. Briefly, paraffin-embedded samples were cut into $4-\mu \mathrm{m}$ sections and placed on polylysine-coated slides. Paraffin sections were baked overnight at $58^{\circ} \mathrm{C}$, deparaffinized in xylene, rehydrated through graded ethanol, quenched for endogenous peroxidase activity in $0.3 \%$ hydrogen peroxide (diluted in methanol; \#216763, Sigma-Aldrich, St. Louis, MO, USA) at $37^{\circ} \mathrm{C}$ for $15 \mathrm{~min}$, and processed for antigen retrieval by high pressure cooking in citrate antigen retrieval solution (pH 6.0; \#MVS-0066, MXB, Fuzhou, Fujian, China) for $\sim 10 \mathrm{~min}$. Sections were incubated at $37^{\circ} \mathrm{C}$ for $1.5 \mathrm{~h}$ with rabbit polyclonal antibodies against $\mathrm{Sp} 1$ (1:3,200, \#07-645, Millipore, Temecula, CA, USA) and HIF1 $\alpha$ (1:200, ab114977, Abcam, Cambridge, MA, USA) in a moist chamber. Immunostaining was performed using the GTVision $^{\mathrm{TM}}$ III Detection System/Mo \& Rb kit (Gene Tech, Shanghai, China), which resulted in a brown-colored precipitate at the antigen site. Subsequently, sections were counterstained with hematoxylin (Sigma-Aldrich) and mounted in a non-aqueous mounting medium. All repetitions included a no primary antibody control. The immunohistochemically stained tissue sections were scored separately by two pathologists blinded to the clinical parameters. The staining intensity was scored as 0 (negative, -), 1 (weak, +), 2 (medium, ++) or 3 (strong, +++). The extent of staining was scored as $(0,<5 \% ; 1,5-25 \% ; 2,26-50 \%$; $3,51-75 \%$; and $4,>75 \%$ ) according to the percentages of the positive staining areas in relation to the whole carcinoma area. Scores for staining intensity and percentage positivity of cells were then multiplied to generate the immunoreactivity score for each case. Tumors with a final immunoreactivity score of $<4$ were considered to be low (-), and those with a score of $\geq 4$ were considered to be high $[4,+; 6,++$; or $\geq 8,+++]$.

Statistical analysis. R 3.1.2 software (Institute for Statistics and Mathematics, Vienna, Austria) was used to organize and process the data downloaded from the TCGA to analyze the correlation between specific gene expression and prognosis. Statistical analyses were performed using SPSS 13.0 (SPSS Inc., Chicago, IL, USA). Student's $t$-test, $\chi^{2}$ test, or Mann-Whitney test, were performed as appropriate. Patients who were alive and did not relapse were censored at the date of their last follow-up visit. Cancer-specific survival (CSS) was defined as the time between the date of initial surgery and the cancer-specific mortality. Survival rates were estimated by the Kaplan-Meier method (13). The hazard ratio (HR) for relationships between each variable and recurrence were calculated 
A
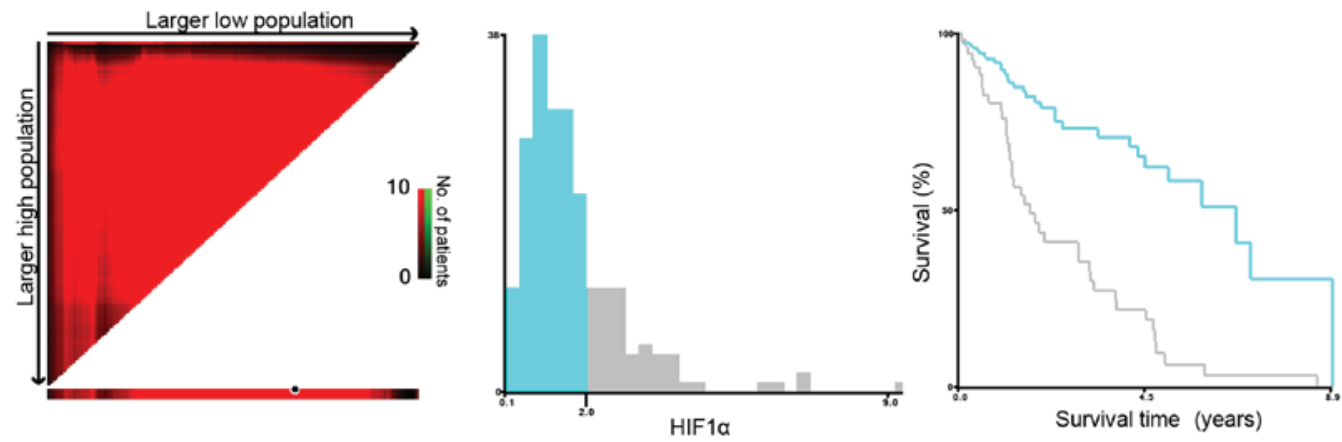

B
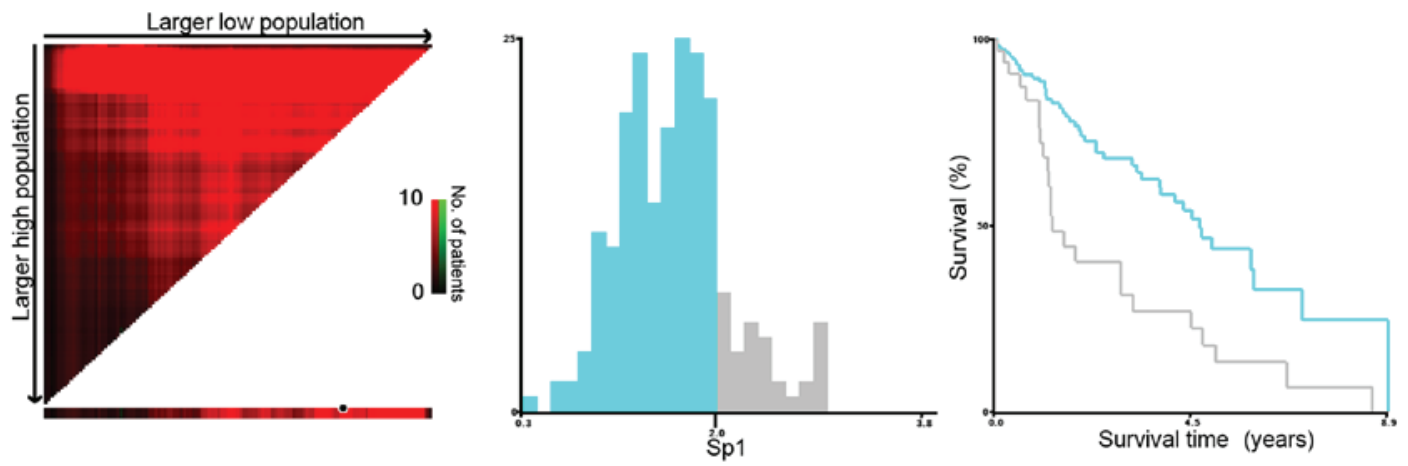

Figure 1. X-tile analysis of survival data for (A) HIF1 $\alpha$ and (B) Sp1 expression levels from The Cancer Genome Atlas registry. X-tile analysis was performed on patient data from the Surveillance, Epidemiology, and End Results Program registry, which was equally divided into training and validation sets. X-tile plots of training sets are presented in the left panels, with plots of matched validation sets shown in the smaller inset. The construction of X-tile plots is more fully described in previous articles (13). The optimal cutoff point highlighted by the black circle in the left panels is shown on a histogram of the entire cohort (middle panels), and a Kaplan-Meier plot (right panels). P-values were determined by using the cutoff point defined in the training set and applying it to the validation set. Figures shows HIF1 $\alpha$ and Sp1 divided at the optimal cutoff point $\left(2, \chi^{2}=116.376, \mathrm{P}<0.001\right)$. HIF1 $\alpha$, hypoxia-inducible factor $1 \alpha$; Sp1, transcription factor specificity protein 1.

using binary Cox regression model (14). Receiver operating characteristic (ROC) curves for CSS prediction were plotted to verify the prediction ability of co-expression of Sp1 and HIF1 $\alpha$ and each single gene. The correlations between Sp1 and HIF1 $\alpha$ expression (scores derived from staining intensity by IHC in HCC specimens) and vital status in HCC patients (succumbed/survived) were analyzed by $\chi^{2}$ test. A P $<0.05$ was considered to indicate a statistically significant difference. All confidence intervals (CIs) were stated at the $95 \%$ confidence level.

\section{Results}

Patient characteristics. A total of 214 eligible patients with hepatic cancer were identified in the TCGA database during the 17-year study period. There were $146(68.2 \%)$ males and 68 (31.8\%) females. The median age was 64 (range, 20-88) years, and the median follow-up period was 414 (range, 10-3,258) days. Patient demographics and clinicopathological features are summarized in Table I.

Clinicopathological differences between the two groups according to vital status. When compared with surviving patients, those patients who succumbed to cancer were more likely to have a relative family cancer history $(\mathrm{P}=0.011)$, to have received a partial incision surgical procedure $(\mathrm{P}=0.025)$, to have an advanced $\mathrm{G} 3 / \mathrm{G} 4$ tumor grade $(\mathrm{P}=0.011)$, and to have an AJCC stage of advanced stage III/IV $(\mathrm{P}=0.042)$. The expression levels of HIF1 $\alpha$ and Sp1 were significantly higher in patients who succumbed to cancer $(\mathrm{P}=0.001)$. However, with regards to other clinicopathological features, no significant differences between the two groups were found (Table I).

Impact of gene expression on survival outcomes in hepatic cancer. The CSS rates were $60.1 \%$ at 3 years (1,067 days), $35.8 \%$ at 5 years (1,823 days) and $9.5 \%$ at 10 years (3,528 days). Given the significant influences of HIF1 $\alpha$ and Sp1 expression levels (as continuous variables) on survival in univariate analysis, the present study attempted to differentiate the patients with a high-risk for cancer mortality by investigating the certain cutoff points of expressions for both of the two genes. The X-tile program (http://www.tissuearray.org/rimmlab/) was applied for the analysis, by identifying the cutoff with the minimum P-values from log-rank $\chi^{2}$ statistics for the categorical value of gene expression in terms of survival (15). X-tile plots were constructed and the maximum of $\chi^{2} \log$-rank values was produced applying both of $2\left(\times 10^{3}\right)$ as cutoff values to divide the cohort into high and low subsets in terms of CSS according to the expressions of Sp1 and HIF1 $\alpha$ (Fig. 1A and B). When the gene expression levels (as binary variables), associated with those independent variables identified by univariate analysis, were included in multivariate Cox regression analysis, only high expression levels of Sp1 and HIF1 $\alpha\left(\geq 2 \times 10^{3}\right)$ were independent predictors for cancer mortality (Table II).

Impact of combination testing for Spl and HIF1 expressions on survival outcomes. When cancer mortality was analyzed as a binary variable, an intermediate AUC for the ROC curve 
Table I. Characteristics of subjects with hepatic cancer from The Cancer Genome Atlas database by vital status.

Vital status

Variables

Male patients

$104(71.2)$

$42(58.5)$

NS

Age at diagnosis (years)

$61.0 \pm 12.0$

$61.0 \pm 13.0$

NS

Height (cm)

$168.5 \pm 9.1$

$166.9 \pm 15.8$

Weight (kg)

$87.9 \pm 111.0$

$88.2 \pm 109.8$

Race

White

69 (48.9)

8 (5.7)

$44(60.3)$

Black or African American

Asian

$59(41.8)$

5 (6.8)

Other

5 (3.5)

$19(26.0)$

5 (6.8)

Ethnicity

Hispanic or Latino

4 (2.8)

Other

137 (97.2)

$4(5.5)$

$69(94.5)$

$41(29.1)$

34 (46.6)

NS

Relative family cancer history

$58(41.1)$

$26(35.6)$

Hepatitis B/C

$17(12.1)$

$10(13.7)$

Alcohol consumption

17 (12.1)

5 (6.8)

None

49 (34.8)

32 (43.8)

$\alpha$-fetoprotein value

$\leq 400 \mathrm{ng} / \mathrm{ml}$

$96(68.1)$

22 (15.6)

23 (16.3)

NS

$>400 \mathrm{ng} / \mathrm{ml}$

Unknown

$23(16.3)$

Surgery

Partial excision $^{\mathrm{a}}$

$90(63.8)$

38 (27.0)

Extended excision ${ }^{\mathrm{b}}$

13 (9.2)

NS

$42(57.5)$

$19(26.0)$

12 (16.4)

0.011

NS

$36(49.3)$

33 (45.2)

$4(5.5)$

Histology diagnosis

Hepatocellular carcinoma

$137(97.2)$

NS

2 (1.4)

$71(97.3)$

Hepatocholangiocarcinoma

2 (1.4)

2 (2.7)

$0(0.0)$

Tumor grade

$\mathrm{G} 1 / 2$

$91(64.5)$

G3/4

$50(35.5)$

34 (46.6)

$39(53.4)$

Vascular tumor invasion

$100(70.9)$

47 (64.4)

32 (22.7)

16 (21.9)

Micro invasion

3 (2.1)

7 (9.6)

$6(4.3)$

0.011

3 (4.1)

$129(91.5)$

$6(4.3)$

$0(0.0)$

6 (4.3)

NS

R0

R1

R2

65 (89.0)

2 (2.7)

1 (1.4)

5 (6.8)

Child classification

Grade 1

$109(77.3)$

$50(68.5)$

Grade 2

$10(7.1)$

7 (9.6)

Unknown

22 (15.6)

16 (21.9) 
Table I. Continued.

\begin{tabular}{|c|c|c|c|}
\hline \multirow[b]{2}{*}{ Variable } & \multicolumn{2}{|c|}{ Vital status } & \multirow[b]{2}{*}{ P-value } \\
\hline & Alive $(\mathrm{n}=141)$ & Cancer mortality $(\mathrm{n}=73)$ & \\
\hline Pathologic tumor stage & & & 0.020 \\
\hline $\mathrm{T} 1 / 2$ & $88(62.4)$ & $36(49.3)$ & \\
\hline $\mathrm{T} 3 / 4$ & $40(35.5)$ & $31(42.5)$ & \\
\hline Unknown & $3(2.1)$ & $6(8.2)$ & \\
\hline Pathologic nodal stage & & & NS \\
\hline No & $98(69.5)$ & $57(78.1)$ & \\
\hline N1 & $1(0.7)$ & $0(0.0)$ & \\
\hline $\mathrm{Nx}$ & $42(29.8)$ & $16(21.9)$ & \\
\hline Distant metastasis & & & NS \\
\hline M0 & 109 (77.3) & $59(80.8)$ & \\
\hline M1 & $2(1.4)$ & $0(0.0)$ & \\
\hline $\mathrm{Mx}$ & $30(21.3)$ & $14(19.2)$ & \\
\hline AJCC 7th stage & & & 0.042 \\
\hline $\mathrm{I} / \mathrm{II}$ & $114(80.9)$ & $49(67.1)$ & \\
\hline III/IV & $19(13.5)$ & $19(26.0)$ & \\
\hline Unknown & $8(5.7)$ & $5(6.8)$ & \\
\hline \multicolumn{4}{|l|}{ Gene expression $\left(\mathrm{x} 10^{3}\right)$} \\
\hline $\mathrm{HIF} 1 \alpha$ & $1.26 \pm 0.65$ & $2.50 \pm 1.66$ & 0.001 \\
\hline Sp1 & $1.51 \pm 0.39$ & $1.78 \pm 0.60$ & 0.001 \\
\hline
\end{tabular}

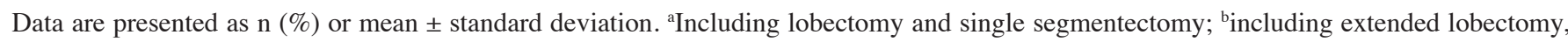
multiple segmentectomy, and total hepatectomy with transplant. AJCC, American Joint Committee on Cancer; NS, no significance; HIF1 $\alpha$, hypoxia-inducible factor $1 \alpha$; Sp1, transcription factor specificity protein 1 .

Table II. Multivariate cox regression analyses for the HIF1 $\alpha$ and Sp1 on cancer-specific survival in hepatic cancer.

\begin{tabular}{lcr}
\hline Independent variable & HR $(95 \% \mathrm{CI})$ & P-value \\
\hline Sex (male vs. female) & $0.802(0.474-1.358)$ & 0.412 \\
Relative family cancer history & $0.953(0.548-1.658)$ & 0.865 \\
Surgery (extended vs. partial excision) & $1.193(0.700-2.035)$ & 0.517 \\
Histology grade (G3/4 vs. G1/2) & $1.332(0.783-2.266)$ & 0.290 \\
Pathologic tumor stage (T3/4 vs. T1/2) & $0.754(0.394-1.444)$ & 0.395 \\
AJCC 7th stage (III/IV vs. I/II) & $1.785(0.836-3.812)$ & 0.134 \\
Sp1 ( $\geq 2,000)$ & $1.907(1.067-3.408)$ & 0.029 \\
HIF1 $\alpha(\geq 2,000)$ & $2.992(1.731-5.171)$ & 0.001 \\
\hline
\end{tabular}

HR, hazard ratio; CI, confidence interval; AJCC, American Joint Committee on Cancer; HIF1 $\alpha$, hypoxia-inducible factor 1 $\alpha$; Sp1, transcription factor specificity protein 1 .

was found to be higher using the combination testing for two genes (0.751) in predicting cancer mortality, compared to either single gene (0.632 for Sp1 and 0.717 for HIF1 $\alpha$ ) (Fig. 2). This indicated that combination testing may be an appropriate and effective approach in the prediction of poor prognosis for hepatic cancer patients. Based on the cutoff points for gene expressions, the samples were divided into 3 groups: G1 (both genes, $<2 \times 10^{3}$ ), G2 (either gene, $\geq 2 \times 10^{3}$ ), and G3 (both genes, $\left.\geq 2 \times 10^{3}\right)$. The risk of cancer mortality increased with high expression of the two genes, and G3 exhibited a greater risk than $\mathrm{G} 2$ when compared to the G1 group ( $\mathrm{HR}=5.420,95 \%$ CI 2.767-10.616, $\mathrm{P}=0.001 ; \mathrm{HR}=3.270,95 \%$ CI 1.843-5.803, $\mathrm{P}=0.001$ ) (Table III, Fig. 3).

Verification by IHC staining. The expression and subcellular localization of Sp1 and HIF1 $\alpha$ in 50 HCC tissues were then determined by IHC. As shown in Fig. 4, Sp1 was expressed predominantly in the nuclei of HCC cells of the tumor regions, 
Table III. Multivariate logistic regression analyses for the combination of Sp1 and HIF1 $\alpha$ on cancer-specific survival in hepatic cancer.

\begin{tabular}{llr}
\hline Independent variable & HR $(95 \%$ CI) & P-value \\
\hline Sex (male vs. female) & $0.786(0.468-1.320)$ & 0.362 \\
Relative family cancer history & $0.939(0.544-1.619)$ & 0.820 \\
Surgery (extended vs. partial excision) & $1.213(0.716-2.055)$ & 0.473 \\
Histology grade (G3/4 vs. G1/2) & $1.428(0.844-2.416)$ & 0.185 \\
Pathologic tumor stage (T3/4 vs. T1/2) & $0.767(0.401-1.467)$ & 0.423 \\
AJCC 7th stage (III/IV vs. I/II) & $1.883(0.882-4.019)$ & 0.102 \\
Expression of Sp1 and HIF1 $\alpha$ & & \\
Both markers $<2,000$ & $1.000($ reference) & 0.001 \\
Either one $\geq 2,000$ & $3.270(1.843-5.803)$ & 0.001 \\
Both markers $\geq 2,000$ & $5.420(2.767-10.616)$ & \\
\hline
\end{tabular}

HR, hazard ratio; CI, confidence interval; AJCC, American Joint Committee on Cancer; HIF1 $\alpha$, hypoxia-inducible factor 1 $\alpha$; Sp1, transcription factor specificity protein 1 .

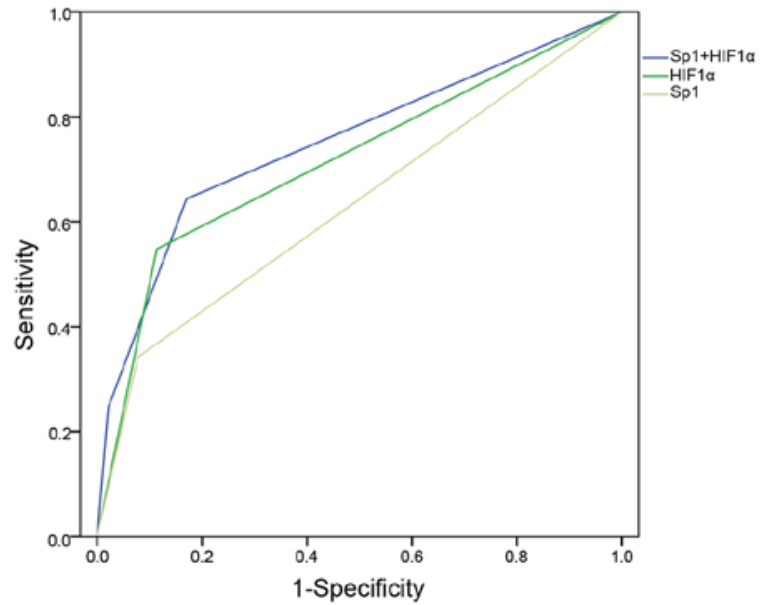

Figure 2. Predictive values of HIF1 $\alpha$ and Sp1 expressions. The combination testing for both gene expressions could better predict the clinical outcomes compared with the single marker. HIF1 $\alpha$, hypoxia-inducible factor $1 \alpha$; Sp1, transcription factor specificity protein 1 .

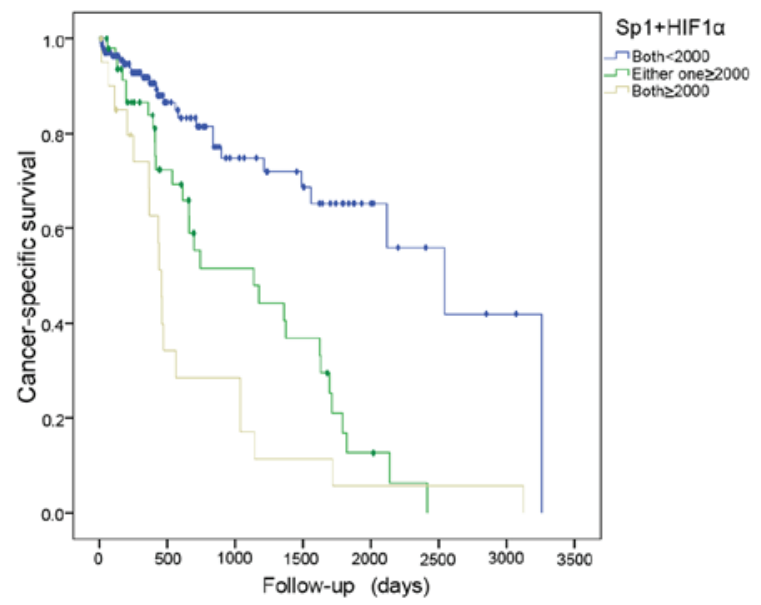

Figure 3. Kaplan Meier curve for cancer-specific survival in 214 samples with hepatic cancer according to the high expressions for HIF1 $\alpha$ and Sp1. (Log Rank test, $\mathrm{P}=0.001)$. HIF1 $\alpha$, hypoxia-inducible factor $1 \alpha$; Sp1, transcription factor specificity protein 1 . and HIF1 $\alpha$ was expressed in the nuclei and plasma. The proportions of Sp1 and HIF1 $\alpha$ expression levels according to each score in 25 patients who survived and the remaining 25 patients who succumbed to cancer are shown in Table IV. As a result, patients who succumbed to HCC presented significantly higher score proportions than the surviving patients for the two genes according to IHC. A total of 15 samples from patients who succumbed to cancer were evaluated as $(+++)$ for Sp1 and HIF1 $\alpha(60.0 \%)$, while none of the patients who survived exhibited such high scores for either gene. This phenomenon emphasized the adverse influence of Sp1 or HIFl $\alpha$ on prognosis in HCC patients.

\section{Discussion}

Using a series of samples from TCGA database, the expression of Sp1 and HIF1 in HCC cancer tissues were analyzed, and it was demonstrated that patients with high expression of either gene had a poorer prognosis, especially in the patients with co-expression.

It is generally accepted that tumor recurrence and metastasis are exclusively associated with tumor angiogenesis following initial therapy (16). Tumor angiogenesis may provide nutrients for the invasion of tumor cells; it also promotes cancer cell transfer to other parts of the body through the blood. Therefore, a biomarker that is associated with angiogenesis in tumor cells may be more objective in predicting the risk of recurrence and metastasis in cancer patients following combined treatment, which may allow timely intervention and action.

In previous studies, the expression levels of Sp1 and HIF1 were found to be crucial in the regulation and formation of blood vessels (17-20). Jia et al (21) reported that Spl serves an important regulatory role in human pancreatic tumor angiogenesis, which was subsequently used as an effective target for anti-angiogenic therapy. Similarly, Seznec et al (22) found that mithramycin competitively inhibits Spl binding and interferes with its transcription in glioma angiogenesis. Meanwhile, HIF1 is a transcription factor widespread in mammals under hypoxic conditions; it mediates cellular adaptation to 
Table IV. The association between Sp1 and HIF1 $\alpha$ according to immunohistochemistry and survival in 50 patients with hepatic cancer.

\begin{tabular}{|c|c|c|c|c|c|c|c|c|c|}
\hline \multirow{3}{*}{$\begin{array}{l}\text { Tumor } \\
\text { tissue }\end{array}$} & \multicolumn{4}{|c|}{ Outcomes $^{\mathrm{a}}$} & \multirow{3}{*}{$\begin{array}{l}\text { Tumor } \\
\text { tissue }\end{array}$} & \multicolumn{4}{|c|}{ Outcomes $^{\mathrm{b}}$} \\
\hline & \multicolumn{2}{|c|}{ Alive } & \multicolumn{2}{|c|}{ Cancer mortality } & & \multicolumn{2}{|c|}{ Alive } & \multicolumn{2}{|c|}{ Cancer mortality } \\
\hline & $\mathrm{N}$ & $\%$ & $\mathrm{~N}$ & $\%$ & & $\mathrm{~N}$ & $\%$ & $\mathrm{~N}$ & $\%$ \\
\hline Sp1 & & & & & HIF1 $\alpha$ & & & & \\
\hline - & 4 & 16.0 & 1 & 4.0 & - & 6 & 24.0 & 1 & 4.0 \\
\hline+ & 15 & 60.0 & 1 & 4.0 & + & 16 & 64.0 & 2 & 8.0 \\
\hline++ & 3 & 12.0 & 5 & 20.0 & ++ & 2 & 8.0 & 4 & 16.0 \\
\hline+++ & 3 & 12.0 & 18 & 72.0 & +++ & 1 & 4.0 & 18 & 72.0 \\
\hline
\end{tabular}

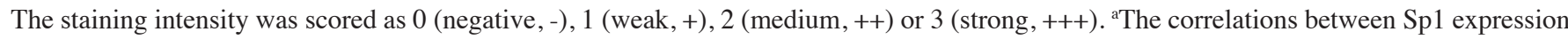

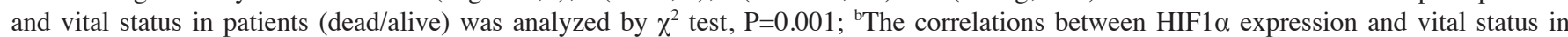
patients (dead/alive) was analyzed by $\chi^{2}$ test, $\mathrm{P}=0.001$. HIF $1 \alpha$, hypoxia-inducible factor $1 \alpha$; Sp1, transcription factor specificity protein 1 .

A

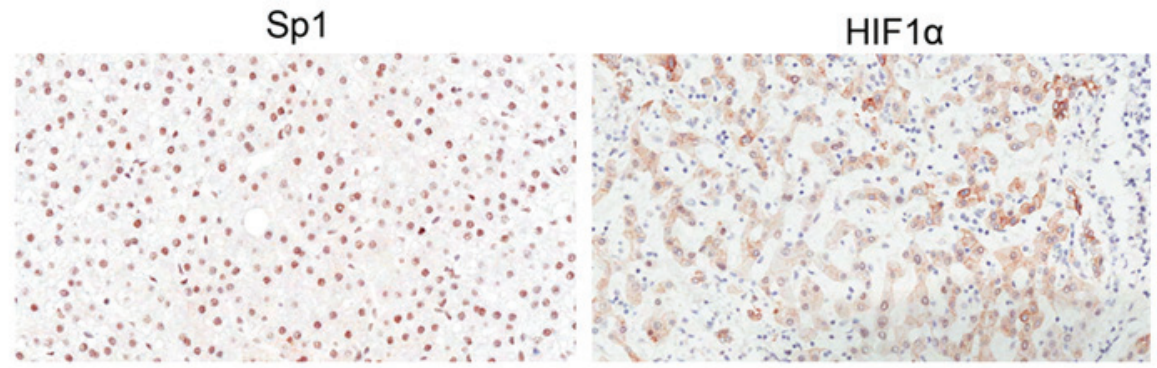

B

Sp1

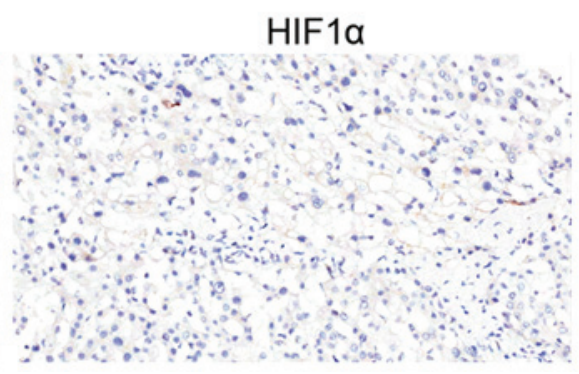

Figure 4. Expression of Sp1 and HIF1 $\alpha$ in situ tumors of HCC patients. Sp1 was expressed predominantly in the nuclei of HCC cells of the tumor regions, and HIF1 $\alpha$ was expressed in the nuclei and plasma. The expression levels of Sp1 and HIF1 $\alpha$ were markedly higher in (A) patients who had succumbed to disease compared with (B) surviving patients. HIF1 $\alpha$, hypoxia-inducible factor $1 \alpha$; Sp1, transcription factor specificity protein 1.

hypoxic microenvironments and regulates the survival of cells in hypoxic conditions via multiple signaling pathways. A number of types of solid tumor have hypoxic microenvironments, which can induce the release of a variety of cytokines promoting angiogenesis, thereby promoting tumor growth and metastasis (23-26). Overexpressed HIF1 protein is present in a variety of malignant tumors, which is closely associated with poor prognosis (27-29). HIF1 also regulates a variety of angiogenesis-associated target genes such as vascular endothelial growth factor (VEGF) expression (30). In the present study, the co-expression of Sp1 and HIF1 were evaluated for predicting prognosis in HCC. According to the findings of the present study, the cutoff value that differentiated high expression of Sp1 and HIF1 $\alpha$ was 2,000; compared with low expression in HCC subjects $(<2,000)$, the patients who expressed these genes at a high level had statistically worse prognosis. These results indicate that patients with high expression of the two genes had a higher risk of mortality compared with patients who expressed one gene at a high level or expressed both genes at a low level. The co-expression of these two genes may enhance tumor angiogenesis, therefore accelerating the invasion and metastasis of tumor cells, resulting in poor prognosis. The IHC staining results indicated that patients who succumbed to cancer exhibited significantly higher expression levels compared to those that survived. Therefore, testing for the combination of two genes may better predict the prognosis of patients with HCC.

In addition, the results of univariate analysis also indicated that family cancer history, less-extensive surgical approach, high tumor grade and stage were adverse prognostic factors. Family genetic factors may be involved in the activation of oncogenes and inactivation of tumor suppressor genes, such 
as deletion of tumor suppressor genes phosphatase and tension homolog gene (PTEN) on chromosome 10, a gene involved in tumor cell growth, adhesion, metastasis, invasion, apoptosis and other processes (31). Genetic predisposition may be important in the process of HCC, and family cancer history may exert a higher susceptibility to liver cancer. In addition, high tumor grade and advanced staging results in severe vascular and lymphatic invasion in patients, and less-extended resection as initial therapy resulted in a high risk of residual tumor. All of the above factors likely promoted intrahepatic metastasis via the portal vein system and affected prognosis adversely.

However, in multivariate analysis, the high co-expression of Sp1 and HIF1 conveyed more risk than any other clinicopathological factor that was significant for poor prognosis in univariate analysis. Notably, previous studies aimed at investigating the association between genes and clinicopathological features in HCC showed that Sp1 and HIF1 were not closely associated with other factors such as age, sex, cirrhosis of the liver, serum AFP and tumor size at the time of diagnosis $(32,33)$. Considering the role of these two genes in the progression of angiogenesis, these results suggested that in HCC patients with high expression of genes at diagnosis, the adverse effect may also promote the recurrence and metastasis after initial therapy, worsening the prognosis. Unfortunately, the insufficient data on relapse in HCC patients from TCGA database limited the analysis on the impact of gene expression for disease-free survival for the present study. Multicenter studies with long-term follow-up are recommended to improve our understanding of the effects of high co-expression of Sp1 and HIF1 on prognosis in HCC patients.

The present study has several potential limitations. First, the TCGA database does not include information regarding the administration of chemotherapy and the quality of surgical care or pathological technique, and all of these factors may affect the multivariate analysis. Second, the TCGA database is a public cancer registry data, so the mechanisms underlying the associations between the gene expression and prognosis may not be further studied.

In summary,the present study demonstrated that high expression levels of Sp1 and HIF1 $\alpha$ in HCC tissues were associated with poor prognosis. In particular, the co-expression of two genes increased the highest risk on cancer mortality. For survival benefit, radical surgery and intense follow-up in the HCC patients with the high co-expression of Sp1 and HIF1a are recommended.

\section{Acknowledgements}

The authors acknowledge the efforts of The Cancer Genome Atlas in the creation of the database. The interpretation and reporting of these data are the sole responsibility of the authors.

\section{References}

1. Tang ZY, Ye SL, Liu YK, Qin LX, Sun HC, Ye QH, Wang L, Zhou J, Qiu SJ, Li Y, et al: A decade's studies on metastasis of hepatocellular carcinoma. J Cancer Res Clin Oncol 130: 187-196, 2004.

2. Gu XQ, Zheng WP, Teng DH, Sun JS and Zheng H: Impact of non-oncological factors on tumor recurrence after liver transplantation in hepatocellular carcinoma patients. World J Gastroenterol 22: 2749-2759, 2016.
3. Briggs MR, Kadonaga JT, Bell SP and Tjian R: Purification and biochemical characterization of the promoter-specific transcription factor, Sp1. Science 234: 47-52, 1986.

4. Ishibashi H, Nakagawa K, Onimaru M, Castellanous EJ, Kaneda Y, Nakashima Y, Shirasuna K and Sueishi K: Sp1 decoy transfected to carcinoma cells suppresses the expression of vascular endothelial growth factor, transforming growth factor beta1, and tissue factor and also cell growth and invasion activities. Cancer Res 60: 6531-6536, 2000.

5. Jiang W, Jin Z, Zhou F, Cui J, Wang L and Wang L: High co-expression of Sp1 and HER-2 is correlated with poor prognosis of gastric cancer patients. Surg Oncol 24: 220-225, 2015.

6. Wang XB, Peng WQ, Yi ZJ, Zhu SL and Gan QH: Expression and prognostic value of transcriptional factor sp1 in breast cancer. Ai Zheng 26: 996-1000, 2007.

7. Yuan P, Wang L, Wei D, Zhang J, Jia Z, Li Q, Le X, Wang H, Yao $J$ and Xie K: Therapeutic inhibition of Sp1 expression in growing tumors by mithramycin a correlates directly with potent antiangiogenic effects on human pancreatic cancer. Cancer 110: 2682-2690, 2007.

8. Pawlus MR and Hu CJ: Enhanceosomes as integrators of hypoxia inducible factor (HIF) and other transcription factors in the hypoxic transcriptional response. Cell Signal 25: 1895-1903, 2013.

9. Lee H, Palm J, Grimes SM and Ji HP: The Cancer Genome Atlas Clinical Explorer: a web and mobile interface for identifying clinical-genomic driver associations. Genome Med 7: 112, 2015.

10. Carbajo-Pescador S, Ordoñez R, Benet M, Jover R, García-Palomo A, Mauriz JL and González-Gallego J: Inhibition of VEGF expression through blockade of Hif $1 \alpha$ and STAT3 signalling mediates the anti-angiogenic effect of melatonin in HepG2 liver cancer cells. Br J Cancer 109: 83-91, 2013.

11. Sobin LH, Gospodarowicz MK and Wittekind C: UICC: TNM Classification of Malignant Tumors. 7th edition. Wiley-Liss, New York, USA, pp1-3, 2009.

12. Edge SB and Compton CC: The American joint committee on cancer: The 7th edition of the AJCC cancer staging manual and the future of TNM. Ann Surg Oncol 17: 1471-1474, 2010.

13. Dinse GE and Lagakos SW: Nonparametric estimation of lifetime and disease onset distributions from incomplete observations. Biometrics 38: 921-932, 1982.

14. Gill RD: Multistate life-tables and regression models. Math Popul Stud 3: 259-276, 1992.

15. Camp RL, Dolled-Filhart M and Rimm DL: X-tile: A new bio-informatics tool for biomarker assessment and outcome-based cut-point optimization. Clin Cancer Res 10: 7252-7259, 2004

16. Cao W, Xu X, Zhang J and Duan Y: Tumor angiogenesis after heated lipiodol infusion via the hepatic artery in a rabbit model of VX2 liver cancer. PLoS One 8: e61583, 2013.

17. Fusté M, Pinacho R, Meléndez-Pérez I, Villalmanzo N, Villalta-Gil V, Haro JM and Ramos B: Reduced expression of SP1 and SP4 transcription factors in peripheral blood mononuclear cells in first-episode psychosis. J Psychiatr Res 47: 1608-1614, 2013.

18. Villa C, Ridolfi E, Fenoglio C, Ghezzi L, Vimercati R, Clerici F, Marcone A, Gallone S, Serpente M, Cantoni C, et al: Expression of the transcription factor Sp1 and its regulatory hsa-miR-29b in peripheral blood mononuclear cells from patients with Alzheimer's disease. J Alzheimers Dis 35: 487-494, 2013.

19. Sun RC and Denko NC: Hypoxic regulation of glutamine metabolism through HIF1 and SIAH2 supports lipid synthesis that is necessary for tumor growth. Cell Metab 19: 285-292, 2014.

20. Chiavarina B, Whitaker-Menezes D, Migneco G, Martinez-Outschoorn UE, Pavlides S, Howell A, Tanowitz HB, Casimiro MC, Wang C, Pestell RG, et al: HIF1-alpha functions as a tumor promoter in cancer associated fibroblasts, and as a tumor suppressor in breast cancer cells: Autophagy drives compartment-specific oncogenesis. Cell Cycle 9: 3534-3551, 2010.

21. Jia Z, Zhang J, Wei D, Wang L, Yuan P, Le X, Li Q, Yao J and Xie K: Molecular basis of the synergistic antiangiogenic activity of bevacizumab and mithramycin A. Cancer Res 67: 4878-4885, 2007.

22. Seznec J, Silkenstedt B and Naumann U: Therapeutic effects of the Sp1 inhibitor mithramycin A in glioblastoma. J Neurooncol 101: 365-377, 2011.

23. McDonald PC, Chafe SC and Dedhar S: Overcoming hypoxia-mediated tumor progression: combinatorial approaches targeting $\mathrm{pH}$ regulation, angiogenesis and immune dysfunction. Front Cell Dev Biol 4: 27, 2016.

24. Badowska-Kozakiewicz AM, Budzik MP and Przybylski J: Hypoxia in breast cancer. Pol J Pathol 66: 337-346, 2015. 
25. Ulivi P, Marisi G and Passardi A: Relationship between hypoxia and response to antiangiogenic therapy in metastatic colorectal cancer. Oncotarget: Apr 12, 2016 (E-pub ahead of print). doi: 10.18632/oncotarget.8712

26. Ye LY, Chen W, Bai XL, Xu XY, Zhang Q, Xia XF, Sun X Li GG, Hu QD, Fu QH and Liang TB: Hypoxia-induced epithelial-to-mesenchymal transition in hepatocellular carcinoma induces an immunosuppressive tumor microenvironment to promote metastasis. Cancer Res 76: 818-830, 2016.

27. Berghoff AS, Ilhan-Mutlu A, Wöhrer A, Hackl M, Widhalm G, Hainfellner JA, Dieckmann K, Melchardt T, Dome B, Heinzl H, et al: Prognostic significance of Ki-67 proliferation index, HIF alpha index and microvascular density in patients with non-small cell lung cancer brain metastases. Strahlenther Onkol 190: 676-685, 2014.

28. Zhao $\mathrm{H}, \mathrm{Wu} \mathrm{Y}$, Chen $\mathrm{Y}$ and Liu H: Clinical significance of hypoxia-inducible factor 1 and VEGF-A in osteosarcoma. Int J Clin Oncol 20: 1233-1243, 2015.
29. dos Santos M, Mercante AM, Louro ID, Goncalves AJ, de Carvalho MB, de Silva EH and da Silva AM: HIF1-alpha expression predicts survival of patients with squamous cell carcinoma of the oral cavity. PLoS One 7: e45228, 2012.

30. Wenger RH: Mammalian oxygen sensing, signalling and gene regulation. J Exp Biol 203: 1253-1263, 2000.

31. Hu TH, Huang CC, Lin PR, Chang HW, Ger LP, Lin YW, Changchien CS, Lee CM and Tai MH: Expression and prognostic role of tumor suppressor gene PTEN/MMAC1/TEP1 in hepatocellular carcinoma. Cancer 97: 1929-1940, 2003.

32. Huang Z, Huang L, Shen S, Li J, Lu H, Mo W, Dang Y, Luo D, Chen $\mathrm{G}$ and Feng Z: Spl cooperates with Sp3 to upregulate MALAT1 expression in human hepatocellular carcinoma. Oncol Rep 34: 2403-2412, 2015.

33. Guo X, Li D, Chen Y, An J, Wang K, Xu Z, Chen Z and Xing J: SNP rs2057482 in HIF1A gene predicts clinical outcome of aggressive hepatocellular carcinoma patients after surgery. Sci Rep 5: 11846, 2015. 